\begin{tabular}{|c|l|}
\hline Title & A llene oxide cyclase is essential for theobroxide induced jasmonic acid biosynthesis in Pharbitis nil \\
\hline Author(s) & Kong, Fanjiang; A be, Jun; Takahashi, Kosaku; Matsuura, Hidey uki; Y oshihara, Teruhiko; Nabeta, Kensuke \\
\hline Citation & $\begin{array}{l}\text { Biochemical and Biophysical Research Communications, 336(4), 1150-1156 } \\
\text { https:/doi.org/10.1016j.bbrc.2005.08.246 }\end{array}$ \\
\hline Issue Date & 2005-11-04 \\
\hline Doc URL & http://hdl.handle.net/2115/8514 \\
\hline Rights & Copyright $\odot$ 2006 Elsevier GmbH. All rights reserved \\
\hline Type & article (author version) \\
\hline File Information & Fanjiang Kong-BBRC.pdf \\
\hline
\end{tabular}

Instructions for use 


\section{Allene oxide cyclase is essential for theobroxide-induced jasmonic acid biosynthesis in Pharbitis nil}

Fanjiang Kong ${ }^{1}$, Jun $\mathrm{Abe}^{2}$, Kosaku Takahashi ${ }^{1}$, Hideyuki Matsuura ${ }^{1}$, Teruhiko Yoshihara $^{1}$ and Kensuke Nabeta ${ }^{1}$

${ }^{1}$ Laboratory of Bioorganic Chemistry, Division of Applied Bioscience, Graduate School

of Agriculture, Hokkaido University, Kita 9, Nishi9, Kita ku, Sapporo, 060-8589, Japan

${ }^{2}$ Laboratory of Plant Genetics \& Evolution, Division of Applied Bioscience, Graduate School of Agriculture, Hokkaido University, Kita 9, Nishi9, Kita ku, Sapporo, 060-8589, Japan

Corresponding author: Kensuke Nabeta

Kita 9, Nishi9, Kita ku, Sapporo, 060-8589, Japan

Phone: +81-11-706-2505

Fax: +81-11-706-2505

E-mail address: knabeta@chem.agr.hokudai.ac.jp 


\section{Abstract}

Theobroxide, a natural product, strongly stimulates the biosynthesis of jasmonic acid (JA) in Pharbitis nil. In this study, we investigated the accumulation of protein by the immunoblot analysis of lipoxygenase (LOX), allene oxide synthase (AOS) and allene oxide cyclase (AOC), key enzymes in JA biosynthesis, and how the endogenous levels of JA in Pharbitis nil are affected by theobroxide. The effect of JA on the accumulations of these proteins was monitored simultaneously. The results show that theobroxide treatment led to a high level accumulation of JA, which is due to high accumulations of LOX, AOS and AOC proteins induced by theobroxide treatment both under short day (SD) and long day (LD) conditions. However, under SD conditions AOS and AOC proteins are not enhanced by JA treatment. Kinetic analysis of protein levels show that a biphasic activation of AOC protein by theobroxide is displayed and the first activation of AOC protein together with elevated JA levels are observed within 30 minutes after treatment. Meanwhile, AOS and LOX proteins are activated by theobroxide later than AOC protein, suggesting that AOC plays an essential role in the initial JA formation induced by theobroxide. Since theobroxide-increased JA levels also show a biphasic manner similar to AOC activation and AOS, LOX proteins are activated later than AOC, and thus we propose a positive JA feedback regulation. 
Interestingly, AOS protein, which is also the enzyme for the biosynthesis of 9,10-ketol-octadecadienoic acid (KODA, a flowering inducing factor), accumulates markedly due to the simultaneous involvement of theobroxide and SD conditions, suggesting that AOS probably plays a role in flower bud formation in Pharbitis nil. Keywords: Allene oxide cyclase; Allene oxide synthase; Jasmonic acid; Lipoxygenase; Pharbitis nil; Theobroxide

Abbreviations: AOC, allene oxide cyclase; AOS, allene oxide synthase; JA, jasmonic acid; KODA, 9,10-ketol-octadecadienoic acid; LOX, lipoxygenase; LD, long day; SD, short day; GC-SIM-MS, gas chromatography selected ion monitoring mass spectrometry.

\section{Introduction}

Theobroxide (Fig 1), a potato tuber inducing compound, was isolated from the culture filtrate of Lasiodiplodia theobromae [1]. Interestingly, spraying theobroxide on the leaflets of potatoes (Solanum tuberosum L.) and the leaves of morning glory (Pharbitis nil), induced potato tuber and flower buds formation, respectively, under non-inductive conditions [2]. Furthermore, theobroxide was shown to inhibit stem elongation in spinach (Spinacia oleracea L.) [3] and morning glory [4, 5]. In morning glory, theobroxide was proven to inhibit stem elongation through suppressing 
gibberellin biosynthesis and stimulating jasmonic acid biosynthesis [4]. Another report also showed that theobroxide strongly induced JA biosynthesis in morning glory [6]. In potato, theobroxide-induced tuber formation is correlated with increased endogenous levels of JA, and elevated LOX activity [7].

JA is synthesized by one of seven different branches of the lipoxygenase (LOX) pathway, which is initiated by the LOX-catalyzed oxygenation of $\alpha$-linolenic acid $(\alpha-\mathrm{LeA})[8]$. Insertion of oxygen can take place at position 13 by a 13-LOX or at position 9 by a 9 -LOX. The 13-LOX product with $\alpha$-LeA as a substrate is a hydroperoxide (13S-hydroperoxy-(9Z,11E,15Z)-octadecatrienoic acid, 13-HPOT) (Fig. 1). 13-HPOT can be metabolized by an allene oxide synthase (AOS) into an unstable allene oxide, which is cyclized by an allene oxide cyclase (AOC) to cis-(+)-OPDA (9S, $13 S$ ) carrying the enantiomeric structure of the naturally occurring JA. The subsequent reduction of the cyclopentenone ring and three steps of $\beta$-oxidation lead to JA (Fig. 1). In morning glory, JA involves the theobroxide-inhibited stem elongation [4], while in potato, theobroxide induces tuber formation by stimulating JA and tuberonic acid (TA, Fig. 1) biosynthesis. Subsequently, JA and TA are then converted into the potato tuber-inducing compound, tuberonic acid glucoside (TAG, Fig 1) [9, 10, 7]. As a flower inducing factor, 9-hydroxy-10-oxo-12(Z),15(Z)-octadecadienoic acid 
(9,10-ketol-octadecadienoic acid, KODA, Fig. 1) was isolated from Lemna paucicostata.

There is a close relationship between flower induction and the transient elevation of the endogenous KODA level during the dark period, suggesting that

KODA may be involved in flower induction in P. nil [11]. KODA is presumed to be formed from 9-hydroperoxy-10(E),12(Z),15(Z)-octadecatrienoic acid (9-HPOT), generated from linolenic acid by 9- LOX and AOS (Fig 1) [12, 13]. Thus, AOS probably plays a role in flower bud formation in P. nil.

Theobroxide strongly induces JA formation and flower bud formation in morning glory, but these mechanisms remain to be elucidated. Therefore, in this study, we investigated the protein accumulations of the enzymes in JA biosynthesis by immunoblot analysis and the changes in the endogenous JA levels. The action mechanisms of theobroxide for inducing JA biosynthesis and flower bud formation will be discussed.

\section{Results}

Organ specificity of LOX, AOS and AOC expression in P. nil

Immunoblot analysis of total proteins, extracted from various organs revealed the abundant appearance of LOX in leaves, cotyledons and flower buds of mature $P$. nil plants. Much less protein occurs in stems and petioles and almost undetectable levels 
could be found in flowers and roots. AOS protein is predominantly found in the leaves and low levels are detected in cotyledons. Unlike in Arabidopsis [14], AOS protein is undetectable in flower organs in P. nil. AOC protein is also observed at high levels in the leaves, less protein occurs in the cotyledons and flower buds and, no protein is found in other organs (Fig. 2). All these results suggested that the leaf is the main site for the biosynthesis of JA in P. nil. Therefore, we selected leaves as the materials for further analysis.

Accumulation of LOX, AOS and AOC proteins and JA levels in P. nil induced by theobroxide

Our previous results $[4,6]$ demonstrated that theobroxide strongly stimulates JA biosynthesis in P. nil. However, the mechanism of enhancement of JA biosynthesis by theobroxide remains unclear. Therefore, in this study we investigated the accumulations of three enzymes in JA the biosynthesis pathway affected by theobroxide via an immunoblot approach. In comparison, the effect of JA on protein accumulation was also evaluated. One week old seedlings of $P$. nil were treated with theobroxide or JA every 2 days until the leaves were harvested. Total proteins were extracted and the accumulation levels of LOX, AOS and AOC were determined; meanwhile, the endogenous levels of JA were also quantified. The immunoblot analysis showed that LOX, AOS and AOC 
proteins accumulated to high levels after 1 and 2 weeks of theobroxide treatment in $P$. nil under LD conditions (Fig. 4). However, under SD conditions, AOS and AOC proteins accumulated markedly due to theobroxide treatment, while LOX protein remained constant (Fig 4a). The corresponding levels of JA affected by theobroxide are shown in Figure 3. The levels of JA increased dramatically after 1 and 2 weeks of theobroxide treatment both under SD and LD conditions. This is in accordance with the increased accumulations of LOX, AOS and AOC proteins, but LOX accumulation was seemingly not necessary for the theobroxide induced JA production under SD conditions since the LOX protein remained unchanged. Similarly, under LD conditions, JA induced high accumulations of LOX, AOS and AOC proteins (Fig 4). Under SD conditions, unlike the effect of theobroxide, JA only induced the accumulation of LOX, while the accumulation of AOS was reduced and the accumulation of AOC was unaffected after 1 and 2 weeks treatment. These results suggest that the effects of theobroxide and JA on the protein accumulations of JA synthesis enzymes are different and a somewhat complicated process was proposed.

AOC is essential for theobroxide-stimulated jasmonic acid biosynthesis in P. nil Theobroxide treatment led to the accumulation of high levels of LOX, AOS, AOC proteins and endogenous JA after 1 and 2 weeks treatment in $P$. nil. How these 
enzymes are regulated by theobroxide remains unclear. Therefore, we investigated the kinetic accumulation of relative proteins treated with theobroxide by the immunoblot approach. The results show how under SD conditions (Fig 5a), in the biphasic activation of AOC protein, the first and second activation are displayed at 30 minutes and 6 hours after theobroxide application, respectively. While LOX protein accumulation was enhanced by theobroxide later ( 1 hour after theobroxide treatment) than AOC (30 minutes after treatment), LOX accumulation decreases gradually (Fig 5a), while AOS accumulation remains almost constant. Conversely, under LD conditions (Fig 5b), the AOC protein accumulation induced by theobroxide showed a biphasic activation manner, but the second activation was earlier ( 2 hours after treatment) than under SD conditions (6 hours after treatment), and remained constant until 24 hours. Unlike that under SD conditions, AOS protein accumulation was enhanced by theobroxide 1 hour after application. In the case of LOX protein accumulation, stimulation occurred within 2 hours after theobroxide treatment, which was late in comparison with the SD conditions ( 1 hour after treatment). It became clear that AOC is the first protein activated by theobroxide, but not AOS and LOX proteins. The quantitative analysis results showed that JA levels were increased by theobroxide 30 minutes after application (Fig 5c) and at this time only the AOC protein is activated (Fig 5b), 
suggesting that the rise of JA level was due to activated AOC accumulation. Similar to the activation pattern of AOC protein, a biphasic rise of the JA level induced by theobroxide was also displayed. After about 6 hours of theobroxide application, the JA levels were increased again and this second rise of the JA levels was attributed to the enhanced LOX, AOS and AOC protein accumulations, suggesting the occurrence of positive JA feedback regulation. The results we obtained here show that the first peak of theobroxide-induced JA accumulation was dependent on the enhancement of AOC protein accumulation directly simulated by theobroxide, while the second peak of JA accumulation was probably due to the feedback activation of LOX, AOS and AOC protein accumulations by the initially elevated JA accumulation.

In order to test the effect of JA on LOX, AOS and AOC accumulation in the short term, we applied JA onto the seedlings under LD conditions and performed immunoblot analysis. Unlike the effect of theobroxide, slight effects of JA on AOS and AOC protein accumulation could be observed. However, LOX protein accumulation was strongly enhanced by JA treatment and this enhancement was earlier (1 hour after treatment) than that of theobroxide ( 2 hours after treatment, Fig $5 b$ ). These results show that the effects on LOX, AOS and AOC protein accumulation of JA and theobroxide are different, suggesting the presence of different effects of JA and theobroxide on JA 
biosynthesis.

Correlations between AOS protein expression and flower bud formation in P. nil

KODA is involved in flower induction in $P$. nil [11] and is synthesized through catalyzing 9-hydroperoxy-10(E),12(Z),15(Z)-octadecatrienoic acid by AOS protein (Fig 1) $[12,13]$, suggesting that AOS protein might be involved in the flower bud formation induced by theobroxide and SD conditions. Our results show that after 2 weeks, flower bud formation was induced by theobroxide in $P$. nil under non-inductive conditions, LD conditions (Fig 7a). At this time, extremely high levels of AOS protein are accumulated by theobroxide application (Fig 4b). Conversely, under flower inductive conditions (SD conditions), more flower buds were formed in theobroxide treated plants compared with the control plants (Fig 7a), which is also closely related to the high accumulation of AOS protein by theobroxide treatment (Fig 4b). Interestingly, AOS proteins accumulated markedly under SD conditions, while AOS proteins were almost undetectable under LD conditions (Fig 7b). This is in accordance with the fact that SD conditions induce flower bud formation in P. nil [15], which was also observed in this experiment (Fig 7a), but no flower buds were found, even after 20 days under LD conditions. Moreover, by applying a JA analogue, methyl jasmonate, flower bud formation was inhibited in $P$. nil under SD conditions $[16,17]$. This inhibition was also 
in agreement with our results that JA reduces flower bud numbers under SD conditions

(Fig 7a). This was in accordance with the immunoblot results (Fig. 4b), the AOS protein levels were markedly reduced by JA treatment under SD conditions. All these results suggest that AOS proteins are closely related to flower bud formation in P. nil.

\section{Discussion}

Theobroxide induces the tuber formation of potato, induces flower bud formation and inhibits stem elongation in P. nil [2, 4]. Theobroxide-induced potato tuber formation and theobroxide-inhibited stem elongation in P. nil are dependent on the stimulation of JA biosynthesis by theobroxide [4, 7]. It is less understood, however, how this JA stimulation is regulated by theobroxide. JA and its methyl ester, collectively named jasmonates, are ubiquitously occurring plant growth regulators. The stress-induced rise of jasmonates is a common phenomenon in all plants analyzed so far. It has been reported several times that following a stimulus such as elicitation [18] or wounding [19], JA levels increase significantly and often in a complex, biphasic manner (for a review see [20]). This is also the case for theobroxide activated JA accumulation in $P$. nil (Fig. 5c). We have shown here that AOC protein expression is first activated by theobroxide following a transient JA burst after 30 minutes and, subsequently a positive JA feedback regulation probably occurs. Later LOX, AOS and AOC protein 
expressions are enhanced, following the second rise of JA levels (Fig. 5). These results show a preferential role of AOC in the regulation of JA biosynthesis, suggesting the AOC is the major control point for theobroxide-induced JA formation. This is in accordance with the previous results that only the accumulation of AOC protein, but not that of LOX and AOS proteins is affected in Arabidopsis mutant opr3, in which JA downstream biosynthesis enzyme, OPDA reductase, was blocked [21]. The AOC-catalyzed step is regarded as the crucial step in jasmonate biosynthesis, because only this enantiomeric form is the substrate for the naturally occurring JA, which is formed after the reduction of OPDA by a specific OPDA reductase and three cycles of $\beta$-oxidation [22].

Regulation of JA biosynthesis is a quite complex process. In Arabidopsis, LOX, AOS and AOC proteins, which are compartmentalized within the chloroplast [14, 23], might function by substrate channeling during basal JA formation, and stimuli may allow substrate accessibility by the relevant enzymes, thereby increasing JA formation. This model was discussed by Froehlich et al. [24] and is in accordance to previous data on 35S::LOX2antisense plants [23], which exhibited a decrease in wound-induced JA formation only, but were still able to form residual levels of JA. Another possibility in the regulation of JA biosynthesis would be an activity control by post-translational 
modifications of the pre-existing enzymes, such as AOC. Such a control would allow the plant to respond rapidly upon wounding or pathogen/insect attack or theobroxide application by the formation of JA, which functions subsequently as a signal in defense gene expression or for feedback regulation of JA biosynthesis enzymes. The functional role of the subsequent transcriptional up-regulation of JA-biosynthetic enzymes remains to be elucidated.

P. nil (violet) is a typical short-day plant, in which flowering is induced when the day length becomes shorter than a critical value. The process is very sensitive; flowering is induced by exposing a seedling to a single 16 hour dark period after cultivation under continuous light [15]. Under non inductive conditions, theobroxide strongly induces flower bud formation in P. nil $[2,7]$. However, how theobroxide induces flower bud formation in $P$. nil remains unclear. KODA reacts with catecholamines to generate products that strongly induce flowering and a close relationship between flower induction and the transient elevation of the endogenous KODA level during the dark period was observed, suggesting that KODA may be involved in flower induction in $P$. nil $[11,25,26]$. AOS catalyzes 9(S)-hydroperoxy-octadecatrienoic acid (9-HPOT) to 9,10-epoxylinolenic acid, a short-lived allene oxide that forms KODA automatically[12, 13] suggesting AOS may 
involve in flower bud formation in $P$. nil. Here, we show that under inductive conditions (SD conditions or LD conditions with theobroxide application), AOS protein is strongly induced (Fig 7b, Fig 4b), which correlates with the flower bud induction (Fig. 7a). Under SD conditions, JA was shown to inhibit flower bud formation (Fig. 7a) [16, 17], which is in accordance with our observations that AOS accumulation is reduced by JA (Fig 4b). All these results suggest that there is a close relationship between AOS protein expression and flower bud formation, indicating that AOS is probably involved in flower bud formation in P. nil.

\section{Materials and Methods}

Plant materials and growing conditions

Seeds of Pharbitis nil cv. Violet (Marutane Seed Co, Kyoto, Japan) were treated with concentrated $\mathrm{H}_{2} \mathrm{SO}_{4}$ for 1 hour, washed with running tap water for another 3 hours, and then soaked in distilled water overnight at $25^{\circ} \mathrm{C}$. The swollen seeds were sown in pots filled with peat moss and perlite $(1: 1 \mathrm{v} / \mathrm{v})$ under LD (18 hour light/6 hour dark) conditions in growth chambers (NK System, Biotron NC 350, Japan) before treatment. The chambers were equipped with 20 slip fluorescent lamps (NEC FL40SEX-N-HG, Japan) to provide white light $\left(150 \mu \mathrm{mol} \mathrm{m} \mathrm{s}^{-1}\right)$, at a temperature of $25{ }^{\circ} \mathrm{C}$ and relative humidity of $60 \%$. Seven-day-old seedlings were treated with different solutions. Water 
and liquid Hyponex (diluted to 500 times with water, Hyponex Japan Co. Ltd., Japan) were applied to the plants at every two 2 days and once a week, respectively.

\section{Plant treatments}

The photoperiods for SD and LD conditions were set in 10L/14D and 18L/6D, respectively. Theobroxide was isolated from the culture filtrate of Lasiodiplodia theobromae [1]. Theobroxide and JA were each dissolved in a small amount of DMSO, and then diluted in water containing $100 \mathrm{ppm}$ Tween 20 to give a concentration of 1 $\mathrm{mM}$. Water containing a small amount of DMSO and $100 \mathrm{ppm}$ Tween 20 was used as a control solution. Five milliliters of theobroxide or JA solution was sprayed onto the leaf surface of each seedling at 2-day intervals after the plants were transferred. Plant tissues were harvested two weeks after spraying, placed immediately in liquid nitrogen and then stored at $-80^{\circ} \mathrm{C}$ until being analyzed. Ten plants were used in each experiment which was repeated at least 3 times.

\section{Scoring of the flowering response}

The timing date of flower buds was scored and the number of flower buds and flowers per plant in P. nil were counted every two days. Ten plants were used for each treatment, and the average number of flower buds per seedling is presented. Each treatment was repeated at least three times. 


\section{Protein extraction}

About $0.1 \mathrm{~g}$ of frozen leaves was homogenized with a polytron in $1 \mathrm{ml}$ of $0.1 \mathrm{M}$ phosphate buffer ( $\mathrm{pH}$ 6.6). The homogenate was centrifuged for $20 \mathrm{~min}$ at $15,000 \mathrm{~g}$ and the supernatant was used as the enzyme source. Protein contents were determined by the Bradford method, using BSA as standard [27].

\section{Electrophoresis and Immunoblot analysis}

Proteins were analyzed in 10\% SDS-polyacrylamide gel according to Laemmli [28]. In the transfer of electrophoretically separated proteins from the gels to nitrocellulose sheets, we followed the method of Towbin et al. [29]. Blots were developed using chemiluminescence reagent (PerkinElmer Life Sciences, Inc.) according to the manufacturer's instructions. Rabbit polyclonal antibodies raised against potato LOX [30], the recombinant AOS of A. thaliana [14] and the recombinant AOC2 of $A$. thaliana [21], were used. Antibodies were used in the following dilutions: anti-LOX, 1: 5000; anti-AOS, 1: 5000; anti-AOC, 1: 5000.

\section{Extraction and quantification of endogenous JA}

The content of JA was analyzed according to the method of Matsuura et al. [31] with small modifications. Plant tissues $(1 \mathrm{~g})$ were ground under liquid nitrogen and suspended in $80 \%$ aq. $\mathrm{MeOH}(10 \mathrm{ml})$ overnight. The mixture obtained was filtered and 
$500 \mathrm{ng}$ deuterium-labeled JA (internal standard) was added. The filtrate was

concentrated under reduced pressure, re-dissolved in $\mathrm{H}_{2} \mathrm{O}$, adjusted to $\mathrm{pH} 2-3$ with $6 \mathrm{~N}$

$\mathrm{HCl}$, and extracted with EtOAc $(10 \mathrm{ml} \times 3)$. The combined organic layers were washed

with saturated $\mathrm{NaHCO}_{3}(10 \mathrm{ml} \times 3)$, adjusted to $\mathrm{pH} 2-3$ with $6 \mathrm{~N} \mathrm{HCl}$, and extracted with

EtOAc $(10 \mathrm{ml} \times 3)$. The combined organic layers were concentrated and the resulting

residue was dissolved in $1 \mathrm{ml}$ of $\mathrm{H}_{2} \mathrm{O}$ and subjected to the cartridge column of Bond

Elut $\mathrm{C}_{18}$ (Varian, CA, U.S.A.). The column was successively washed with $\mathrm{H}_{2} \mathrm{O}(1 \mathrm{ml} \times 2)$

and $\mathrm{MeOH} / \mathrm{H}_{2} \mathrm{O}(4: 1, \mathrm{v} / \mathrm{v}, 1 \mathrm{ml} \times 4)$. The $80 \% \mathrm{MeOH} / \mathrm{H}_{2} \mathrm{O}$ eluant was concentrated in

vacuo, and the residue was subjected to Bond Elut DEA(Varian, CA, U.S.A.). The

column was successively washed with $\mathrm{MeOH}(1 \mathrm{ml} \times 2)$ and $1 \mathrm{~N} \mathrm{AcOH} / \mathrm{MeOH}(1 \mathrm{ml} \times 4)$.

The $\mathrm{AcOH} / \mathrm{MeOH}$ eluant was evaporated. The residue was dissolved in $\mathrm{MeOH}$,

methylated by ethereal diazomethane and purified by HPLC (Hitachi L7100, HITACHI, Japan) equipped with a column of YMC-Pack ODS (300 mm×10 mm; YMC Co, Ltd., Japan) to give a JA fraction. The fraction was analyzed by a GC- SIM-MS (QP5000 system, SHIMAZU, Japan) using a ZB-1 column $(30 \mathrm{~m} \times 0.25 \mathrm{~mm}, 0.5 \mu \mathrm{m}$ phase thickness). The $\mathrm{GC}$ temperature program was $80^{\circ} \mathrm{C}$ for $1 \mathrm{~min}, 80-290{ }^{\circ} \mathrm{C}$ at $20^{\circ} \mathrm{C} / \mathrm{min}$ and $290{ }^{\circ} \mathrm{C}$ for $5 \mathrm{~min}$. Injector and detector temperatures were 200 and $280{ }^{\circ} \mathrm{C}$, respectively. The amounts of endogenous JA were calculated from the peak areas of JA 
in comparison with the corresponding internal standard.

\section{Acknowledgements}

We thank Dr. C. Wasternack for the supply of anti-AOC-antibodies, Dr. F. Schaller and Prof. E.W.Weiler for the supply of anti-AOS-antibodies, Prof. J. J. Sanchez-Serrano for anti-LOX-antibodies, Mr. Li Li, Dr. Baohui Liu and Dr. Shinya Mitsuhashi for their help with immunoblot analysis. Dr. C. Wasternack and Dr. F. Schaller are gratefully acknowledged for their critical readings of the manuscript and helpful suggestions.

\section{References}

[1] K. Nakamori, H. Matsuura, T.Yoshihara, A. Ichihara, Y. Koda, Potato micro-tuber inducing substances form Lasiodiplodia theobromae, Phytochemistry 35 (1994) 835- 839 .

[2] T. Yoshihara, F. Ohmori, K. Nakamori, M. Amanuma, T. Tsutsumi, A. Ichihara, H. Matsuura, Induction of plant tubers and flower buds under noninducing photoperiod conditions by a natural product, theobroxide, J. Plant Growth Regul. 19 (2000) 457-461.

[3] F. Kong, X. Gao, K. H. Nam, K. Takahashi, H. Matsuura T. Yoshihara, Inhibition of stem elongation in spinach by theobroxide, J. Plant Physiol. (2005) in press.

[4] F. Kong, X. Gao, K. H. Nam, K. Takahashi, H. Matsuura T. Yoshihara, Theobroxide 
inhibits stem elongation in Pharbitis nil by regulating jasmonic acid and gibberellin biosynthesis, Plant Sci. (2005) in press.

[5] X. Gao, F. Kong, F. Wang, H. Matsuura, T. Yoshihara, Inhibitory role of gibberellins in theobroxide-induced flowering of Pharbitis nil, J. Plant Physiol. (2005) in press.

[6] Q. Yang, X. Gao, Y. Fujino, H. Matsuura, T. Yoshihara, Effects of theobroxide, a natural product, on the level of endogenous jasmonoids, Z. Naturforsch 59 (2004) 828-834.

[7] X. Gao, Q. Yang, C. Minami, H. Matsuura, A. Kimura, T. Yoshihara, Inhibitory effect of salicylhydroxamic acid on theobroxide-induced potato tuber formation, Plant Sci. 165 (2003) 993-999.

[8] I. Feussner, C. Wasternack, The lipoxygenase pathway, Annu. Rev. Plant Biol. 53 (2002) 275-297.

[9] T. Yoshihara, E-L.A. Omer, H. Koshino, S. Sakamura, Y. Kikuta, Y. Koda, Structure of a tuber-inducing stimulus from potato leaves (Solanum tuberosum L.), Agric. Biol. Chem. 53 (1989) 2835-2837.

[10] T. Yoshihara, M. Amanuma, T. Tsutsumi, Y. Okumura, H. Matsuura, A. Ichihara, Metabolism and transport of $\left[2-{ }^{14} \mathrm{C}\right]( \pm)$ jasmonic acid in potato plant, Plant Cell 
Physiol. 37 (1996) 586-590.

[11] M. Suzuki, S. Yamaguchi, T. Iida, I. Hashimoto, H. Teranishi, M. Mizoguchi, F. Yano, Y. Todoroki, N. Watanabe, M. Yokoyama, Endogenous $\alpha$-ketol linolenic acid levels in short day-induced cotyledons are closely related to flower induction in Pharbitis nil, Plant Cell Physiol. 44 (2003) 35-43.

[12] B.A. Vick, D.C. Zimmerman, Oxidative systems for modification of fatty acids: The lipoxygenase pathway. In The Biochemistry of Plants, A Comprehensive Treatise, Vol. 9. Edited by Stumpf, P.K. and Conn, E.E. pp. 53-90. Academic Press, New York, 1987

[13] M. Hamberg, New cyclopentenone fatty acids formed from linoleic and linolenic acids in potato, Lipids 35 (2000) 353-363.

[14] D. Laudert, E.W. Weiler, Allene oxide synthase: a major control point in Arabidopsis thaliana octadecanoid signaling, Plant J. 15 (1998) 675-684.

[15] S. Imamura, A. Takimoto, Photoperiodic responses in Japanese morning glory, Phabitis nil Chois., a sensitive short day plant, Bot. Mag. Tokyo 68 (1955)235-241.

[16] B. Maciejewska, J. Kopcewicz, Inhibitory effect of methyl jasmonate on flowering and elongation growth in Pharbitis nil, J. Plant Growth Regul. 21 (2002) 216-223.

[17] B. Maciejewska, J. Kesy, M. Zielinska, J. Kopcewicz, Jasmonates inhibit flowering 
in short-day plant Pharbitis nil, Plant Growth Regul. 43 (2004) 1-8.

[18] S. Parchmann, H. Gundlach, M.J. Mueller, Induction of 12-oxo-phytodienoic acid in wounded plants and elicited plant cell cultures, Plant Physiol. 115 (1997) 1057-1064.

[19] T. Albrecht, A. Kehlen, K. Stanl, H-D. Knofel, G. Sembdner, E.W. Weiler, Quantification of rapid transient increases in jasmonic acid in wounded plants using a monoclonal antibody, Planta 191 (1993) 86-94.

[20] E.E. Farmer, Fatty acid signaling in plants and their associated microorganisms, Plant Mol. Biol. 26 (1994) 1423-1437.

[21] I. Stenzel, B. Hause, O. Miersch, R. Kramell, T. Kurz, H. Maucher, H. Weichert, J. Ziegler, I. Feussner, C. Wasternack, Jasmonate biosynthesis and the allene oxide cyclase family of Arabidopsis thaliana, Plant Mol. Biol. 51 (2003) 895-911.

[22] F. Schaller, C. Biesgen, C. Mussig, T. Altmann, E.W. Weiler, 12-Oxophytodienoate reductase 3 (OPR3) is the isoenzyme involved in jasmonate biosynthesis, Planta 210 (2000) 979-984.

[23] E. Bell, R.A. Creelman, J.E. Mullet, A chloroplast lipoxygenase is required for wound-induced jasmonic acid accumulation in Arabidopsis, Proc. Natl. Acad. Sci. U.S.A. 92 (1995) 8675-8679. 
[24] J.E. Froehlich, A. Itoh, G.A. Howe, Tomato allene oxide synthase and fatty acid hydroperoxide lyase, two cytochrome P450s involved in oxylipin metabolism, are targeted to different membranes of chloroplast envelope, Plant Physiol. 125 (2001) 306-317.

[25] M. Yokoyama, S. Yamaguchi, S. Inomata, K. Komatsu, S. Yoshida, T. Iida, Y. Yokokawa, M. Yamaguchi, S. Kaihara, A. Takimoto, Stressinduced factor involved in flower formation of Lemna is an $\alpha$-ketol derivative of linolenic acid, Plant Cell Physiol. 41 (2000) 110-113.

[26] S. Yamaguchi, M. Yokoyama, T. Iida, M. Okai, O. Tanaka, A. Takimoto, Identification of a component that induces flowering of Lemna in the products of the reaction between $\alpha$-ketol linolenic acid (FIF) and norepinephrine, Plant Cell Physiol. $42(2001)$ 1201-1209.

[27] M. M. Bradford, A rapid and sensitive method for the quantification of microgram quantities of protein utilizing the principle of protein-dye binding, Analytic. Biochem. 72: (1976) 248-254.

[28] U.K. Laemmi, Cleavage of structural proteins during the assembly of the head of bacteriophage T4, Nature 227: (1970) 680-685.

[29] H. Towbin, T. Staehelin, J. Gordon, Electrophoretic transfer of proteins from 
polyacrylamide gels to nitrocellulose sheets: procedure and some applications, Proc. Natl Acad. Sci. USA 76: (1979) 4350-4354.

[30] J. Royo, G. Vancanneyt, A.G. Perez, C. Sanz, K. Stormann, S. Rosahl, J.J. Sanchez-Serrano, Characterization of three potato lipoxygenase with distinct enzymatic activities and different organ-specific and wound-regulated expression patterns, J. Biol. Chem. 271 (1996) 21012-21019.

[31] H. Matsuura, F. Ohmori, M. Kobayashi, A. Sakurai, T. Yoshihara, Qualitative and quantitative analysis of endogenous jasmonoids in potato plant, Biosci. Biotech. Biochem. 56 (2002) 1329-1330. 
Figure legends

Figure 1. Chemical structure of theobroxide and putative linolenic acid cascade.

Figure 2. Immunoblot showing the relative abundance of LOX, AOS and AOC proteins in different organs of 3-week-old P. nil plants growing under SD conditions. A total of $20 \mu \mathrm{g}$ protein was applied to each lane. Immunoblots were probed with anti-LOX antibodies, with anti-AOS antibodies and anti-AOC antibodies all at dilution of 1:5000, respectively.

Figure 3. The effect of theobroxide on endogenous JA levels of leaves growing under SD conditions (A) and LD conditions (B). One-week-old seedlings were subjected to theobroxide treatment every 2 days, leaf samples were collected 1 and 2 weeks after treatment started. Data are the means \pm s.d. of $n=3$ independent experiments.

Figrue 4. Immunoblot analysis of LOX (A), AOS (B) and AOC (C) proteins in P. nil following treatment with theobroxide and JA. One-week-old seedlings were subjected to theobroxide and JA treatment every 2 days, leaf samples were collected 1 and 2 weeks after treatment started. Cont, plants were treated with $\mathrm{H}_{2} \mathrm{O}$; Theo, plants were 
treated with theobroxide; JA, plants were treated with JA. A total of $20 \mu \mathrm{g}$ protein was applied to each lane. The dilution of antibodies was described at figure 2.

Figure 5. Kinetics of LOX, AOS and AOC proteins levels of $P$. nil growing under SD (A) and LD (B) conditions or endogenous levels of JA following treatment with Theobroxide. Two-week-old seedlings were subjected to theobroxide treatment, leaf samples were collected at the times indicated. For immunoblot analysis, a total of $20 \mu \mathrm{g}$ protein was applied to each lane. The dilution of antibodies is described in figure 2. For JA quantification (C), identical samples as in (B) were used for JA extraction. Data are the means \pm s.d. of $n=3$ independent experiments.

Figure 6. Kinetics of LOX, AOS and AOC proteins levels of $P$. nil growing under LD conditions following treatment with JA. Two-week-old seedlings were subjected to JA treatment, leaf samples were collected at the indicated times. A total of $20 \mu \mathrm{g}$ protein was applied to each lane. The dilution of antibodies was described at figure 2 .

Figure 7. Numbers of flower buds in P. nil (A) and immunoblot analysis of AOS protein under different photoperiods (B). For (A), one-week-old seedlings were subjected to 
theobroxide and JA treatment every 2 days. The number of flower buds was counted at the indicated time. SD-Cont, plants treated with water under SD conditions; SD-Theo, plants treated with theobroxide under SD conditions; SD-JA, plants treated with JA under SD conditions. For (B), one-week-old seedlings were growing under SD or LD conditions, after 1 and 2 weeks, plant leaves were collected and proteins were isolated. A total of $20 \mu \mathrm{g}$ protein was applied to each lane. Immunoblots were probed with anti-AOS antibodies at a dilution of 1:5000. 


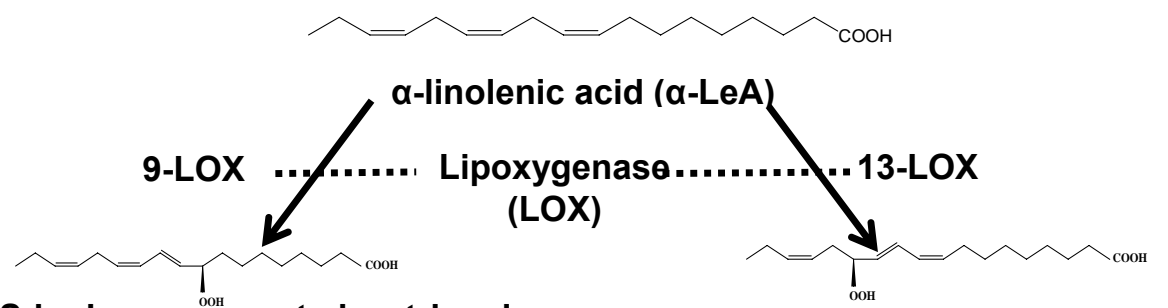

9S-hydroperoxy-octadecatrienoic acid (9-HPOT)

13S-hydroperoxy-octadecatrienoic acid (13-HPOT)
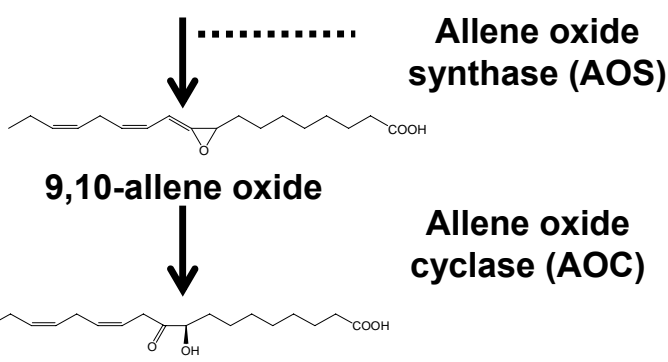

Allene oxide
cyclase (AOC)

9, 10-ketol-octadecadienoic acid (KODA, flower inducing factor)<smiles>CC1=C[C@@H](O)[C@H]2O[C@H]2[C@H]1O</smiles>

Theobroxide

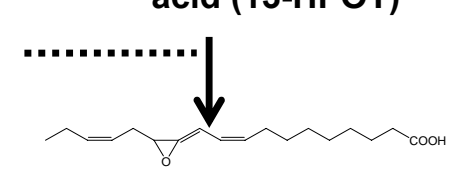

\section{2,13-allene oxide}

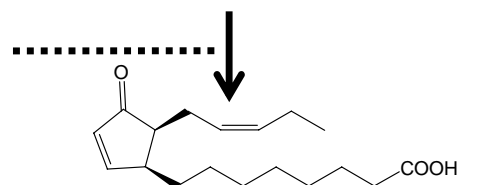

cis-(+)-12-oxo-phytodienoic acid

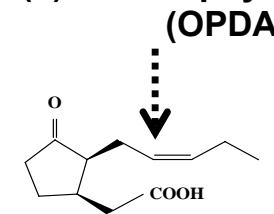

Jasmonic acid (JA)

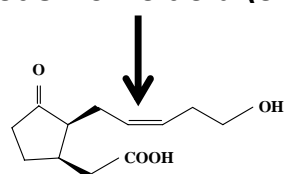

Tuberonic acid (TA)

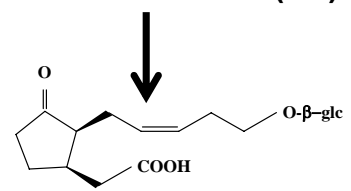

Tuberonic acid glucoside (TAG, potato tuber inducing substance)

Figure 1 


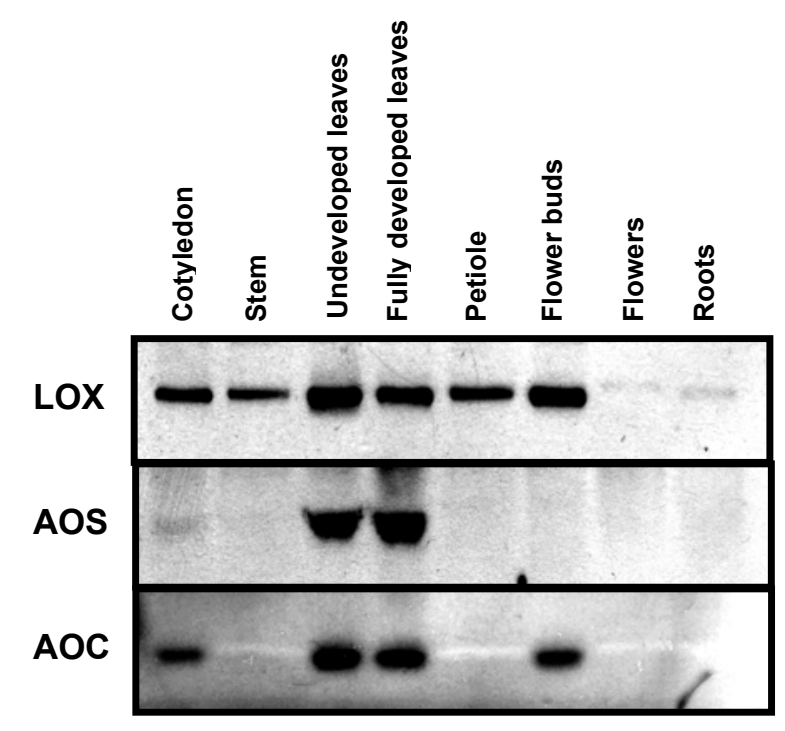

Figure 2 


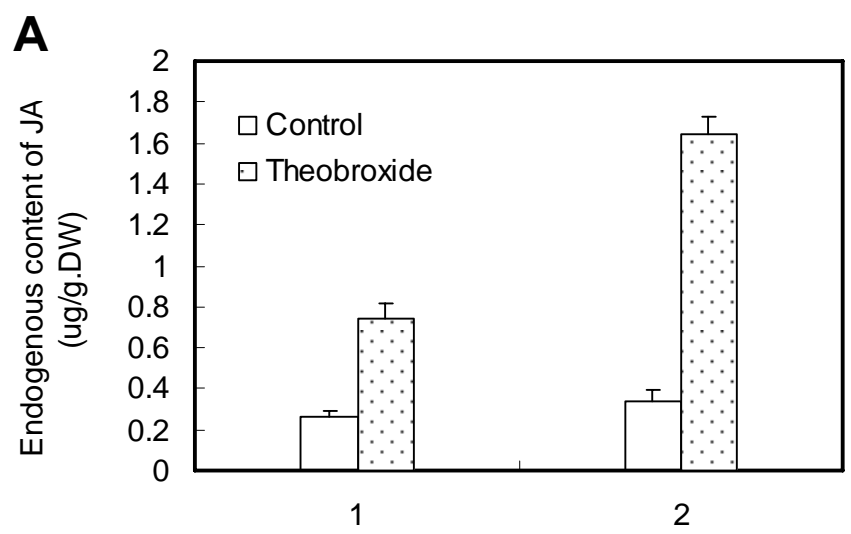

Weeks after chemical treatment

B

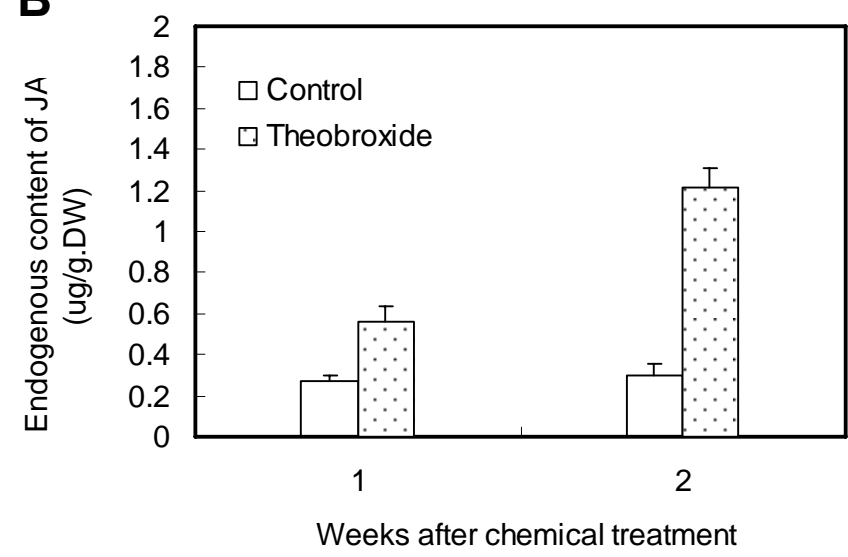

Figure 3 


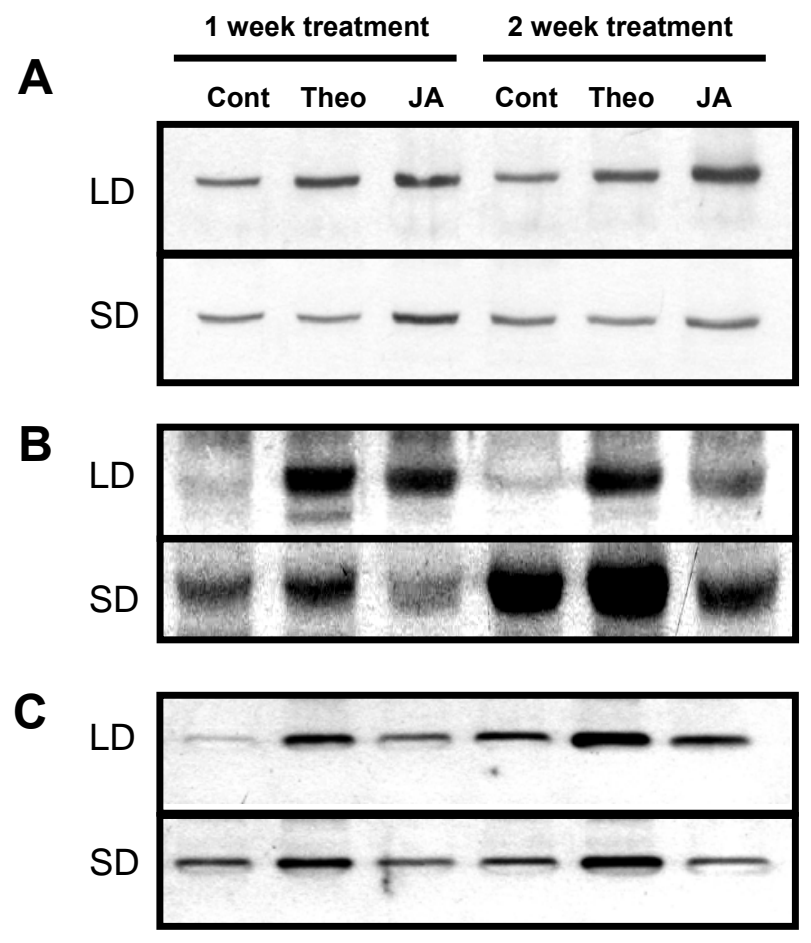

Figure 4 

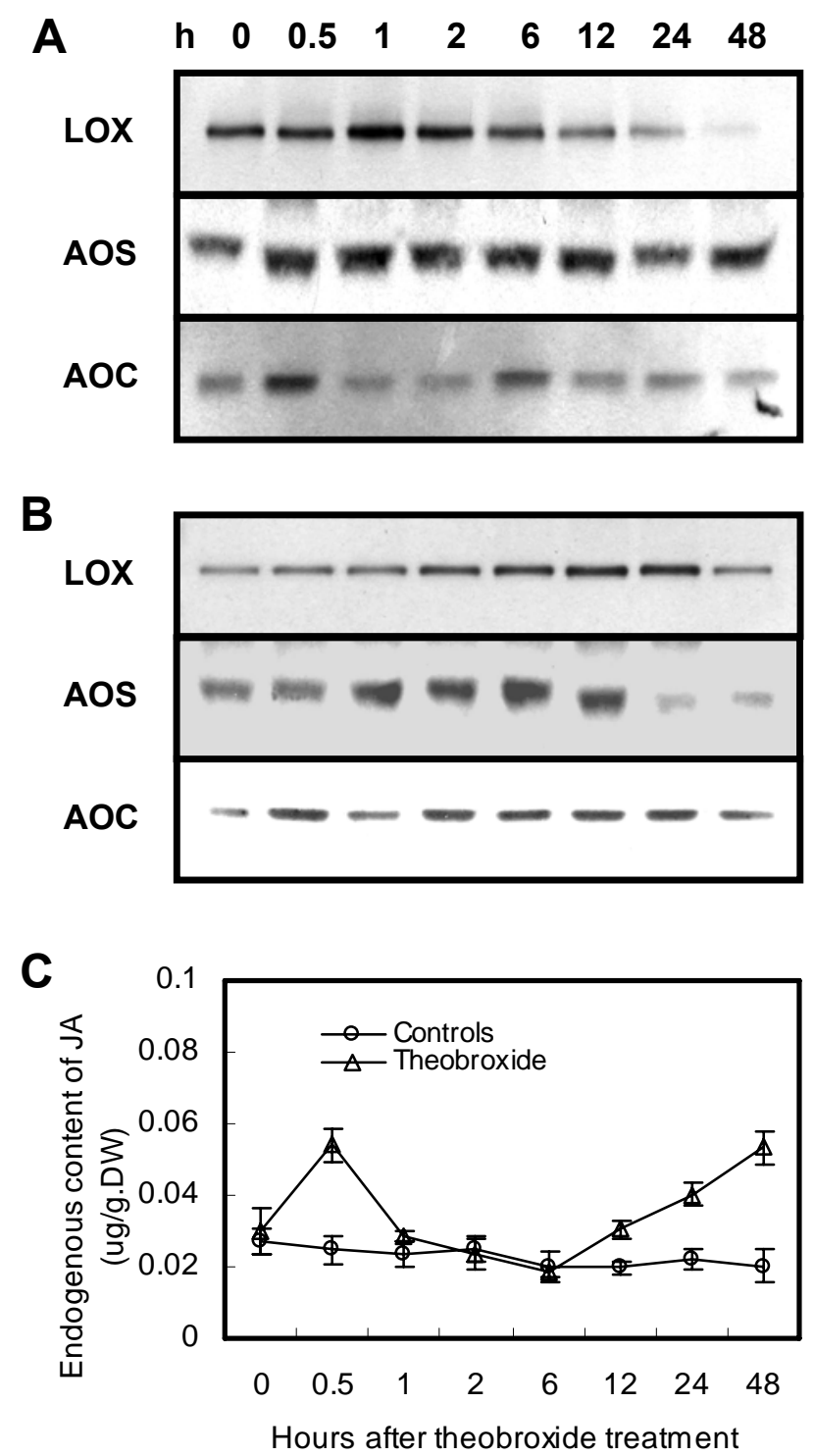

Figure 5 


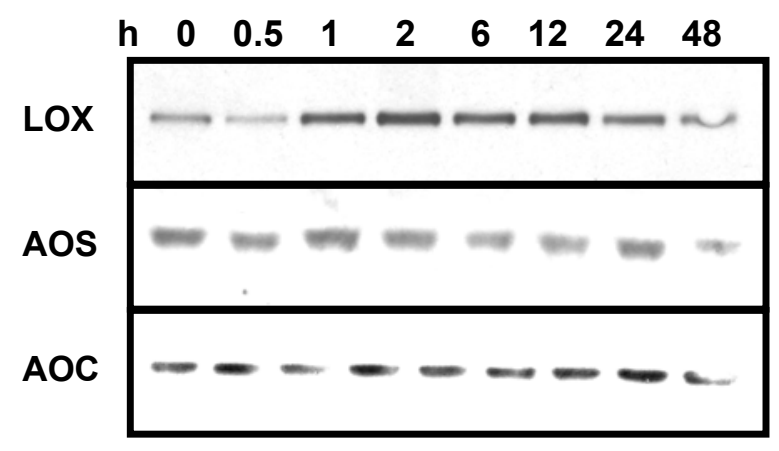

Figure 6 


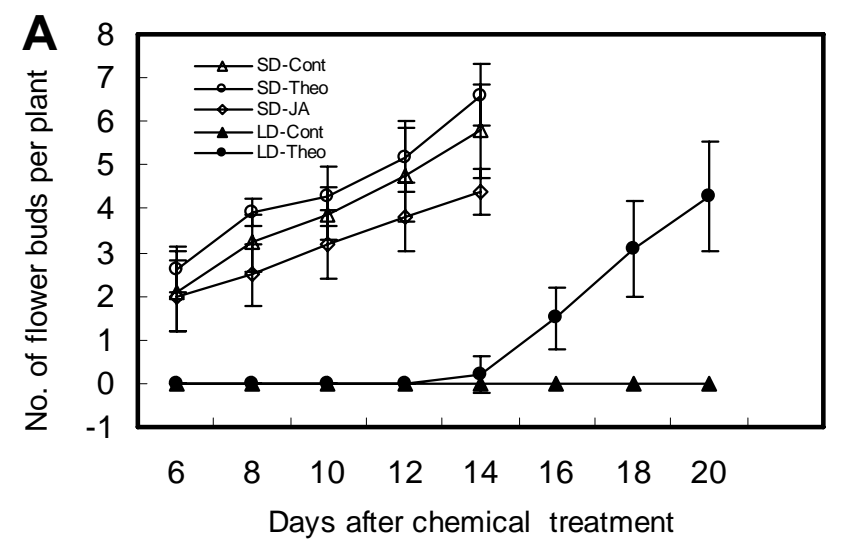

B

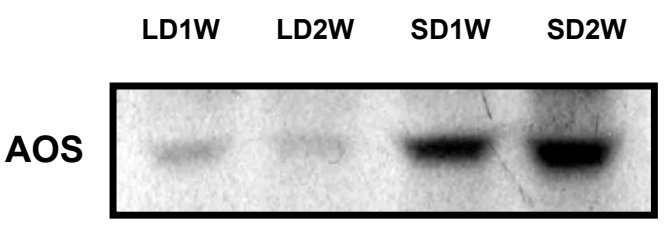

Figure 7 